\title{
Téoros
}

Revue de recherche en tourisme

\section{Changements et évolutions des pratiques professionnelles des prestataires touristiques de sports de nature}

\section{Clémence Perrin-Malterre}

Volume 31, numéro 1, 2012

URI : https://id.erudit.org/iderudit/1020714ar

DOI : https://doi.org/10.7202/1020714ar

Aller au sommaire du numéro

Éditeur(s)

Université du Québec à Montréal

ISSN

0712-8657 (imprimé)

1923-2705 (numérique)

Découvrir la revue

Citer cet article

Perrin-Malterre, C. (2012). Changements et évolutions des pratiques professionnelles des prestataires touristiques de sports de nature. Téoros,

31(1), 115-123. https://doi.org/10.7202/1020714ar
Résumé de l'article

Cet article s'intéresse aux changements qui touchent les pratiques professionnelles des prestataires touristiques des sports de nature en lien avec les évolutions socioculturelles qui concernent la société française, et notamment la montée de la transmodernité. Il se situe dans une approche qui privilégie les logiques d'action des acteurs de l'offre. Pour cette étude qualitative, 10 prestataires sportifs du Puy-de-Dôme ont été interrogés. Les résultats montrent que ces prestataires ont fait de leur passion pour les sports de nature une profession. Ils proposent des pratiques sportives douces axées sur la découverte de l'environnement proche. Dans le cadre de leur prestation, ils souhaitent redonner du sens et de la valeur à l'accueil et à l'échange avec le client. Ils ont fait le choix d'un développement limité et ils font preuve d'un fort attachement au lieu. Ils s'inscrivent ainsi dans un autre projet professionnel que les techniciens du sport et les offreurs de services sportifs.
Ce document est protégé par la loi sur le droit d'auteur. L’utilisation des services d’Érudit (y compris la reproduction) est assujettie à sa politique d'utilisation que vous pouvez consulter en ligne.

https://apropos.erudit.org/fr/usagers/politique-dutilisation/ 


\title{
Changements et évolutions des pratiques professionnelles des prestataires touristiques de sports de nature
}

\author{
Clémence PERRIN-MALTERRE \\ Maître de conférences \\ Université Blaise Pascal \\ clemence.perrin@univ-bpclermont.fr
}

\begin{abstract}
RÉSUMÉ: Cet article s'intéresse aux changements qui touchent les pratiques professionnelles des prestataires touristiques des sports de nature en lien avec les évolutions socioculturelles qui concernent la société française, et notamment la montée de la transmodernité. II se situe dans une approche qui privilégie les logiques d'action des acteurs de l'offre. Pour cette étude qualitative, 10 prestataires sportifs du Puy-de-Dôme ont été interrogés. Les résultats montrent que ces prestataires ont fait de leur passion pour les sports de nature une profession. Ils proposent des pratiques sportives douces axées sur la découverte de l'environnement proche. Dans le cadre de leur prestation, ils souhaitent redonner du sens et de la valeur à l'accueil et à l'échange avec le client. Ils ont fait le choix d'un développement limité et ils font preuve d'un fort attachement au lieu. Ils s'inscrivent ainsi dans un autre projet professionnel que les techniciens du sport et les offreurs de services sportifs.
\end{abstract}

Mots-clés: Sport, nature, changement, transmodernité, logiques d'action.

Depuis l'apparition des sports modernes au XIX ${ }^{\mathrm{e}}$ siècle, des changements culturels sont observables dans la manière de pratiquer les sports de nature (Corneloup, 2009). Le vocable «sports de nature» regroupe une diversité d'activités physiques et sportives terrestres, aquatiques et aériennes qui ont comme point commun de se pratiquer en milieu naturel. Selon l'article L311-1 du Code du sport de France, «les sports de nature s'exercent dans des espaces ou sur des sites et itinéraires qui peuvent comprendre des voies, des terrains et des souterrains du domaine public ou privé des collectivités publiques ou appartenant à des propriétaires privés, ainsi que des cours d'eau domaniaux ou non domaniaux». Au cours du $\mathrm{XX}^{\mathrm{e}}$ siècle, ils sont liés à une culture énergétique du corps dont le pratiquant puise les ressources pour se dépasser et vaincre l'épreuve, et à une culture prométhéenne avec la volonté de devenir «maître et possesseur de la nature» qui s'exprime dans la conquête des espaces naturels. Une forte sociabilité masculine, urbaine et élitiste se développe autour des clubs et des institutions nationales. Les années 1980-2000 marquent l'émergence d'une nouvelle culture postmoderne en rupture radicale avec la culture moderne. Les sports de nature sont alors marqués par des valeurs de liberté, de plaisir et d'amusement.
Une ouverture sociale se produit en direction des jeunes, des femmes, des familles et de différents segments de clientèle en émergence. Actuellement, la culture transmoderne participerait à la reconfiguration des pratiques récréatives de nature (Corneloup, 2011). Les pratiquants seraient à la recherche d'activités où le vertige corporel et émotionnel n'est plus une fin en soi. Les sportifs sont demandeurs d'une relation plus profonde avec la nature où la connaissance du lieu ou encore l'approche patrimoniale occupe une place importante.

Parallèlement à ces changements socioculturels qui touchent les pratiques sportives de nature, l'apparition du concept de développement durable (Charles et Kalaora, 2007), porté par une prise de conscience environnementale et sociale en progression, participe à la remise en cause des modèles de développement touristique moderne, marchandisant les espaces (Girard, 2009). À l'échelle des territoires de montagne, de nouveaux modes de tourisme apparaissent ou se confirment (tourisme "vert», itinérant, etc.), à la fois opérant et découlant d'une redéfinition des valeurs et référentiels culturels. En témoigne par exemple la tendance à un retour vers des stations de sports d'hiver plus petites, moins chères, souvent plus proches, ayant une " ambiance» particulière que l'on ne retrouve 
pas dans les conceptions standardisées de certains grands complexes touristiques de montagne (Bourdeau, 2007).

Il s'agit alors de comprendre comment ces changements issus de transformations sociétales affectent les pratiques professionnelles des prestataires sportifs. Assiste-t-on à l'émergence d'une nouvelle catégorie de professionnels des métiers sportifs de la nature? Quelles sont les caractéristiques et les manières d'exercer le métier de ces prestataires?

Le cadre d'analyse choisi pour répondre à cette question est adapté de la sociologie des logiques d'action proposée par Amblard et al. (1996), cadre d'analyse qui s'inspire de certaines grilles de lecture développées en sociologie des organisations. Dans la tradition de ce courant théorique, nous avons adopté une méthodologie compréhensive en accordant une grande importance à la parole des acteurs recueillie lors des entretiens. Il s'agit donc d'une étude qualitative qui a été menée auprès de 10 prestataires sportifs du département du Puy-de-Dôme.

\section{Cadre théorique}

Plusieurs travaux de recherche se sont intéressés aux spécificités du travail dans le secteur du tourisme sportif. Paget et al. (2007) montrent que ce secteur est composé d'entreprises de petite taille dans lesquelles la spécialisation du travail est faible. C'est également un secteur marqué par une forte saisonnalité des activités, ce qui demande aux professionnels un engagement considérable dans le travail, notamment lors des périodes de grande affluence (Paget et Mounet, 2010). Il s'agit aussi d'un secteur professionnel à la frontière du loisir et du travail, avec pour conséquence un brouillage des temps sociaux (Rech et Paget, 2012). Enfin, la plupart des professionnels du secteur effectuent leur activité dans le cadre d'une passion pour les activités sportives (Bouhaouala, 2008), ce qui demande au prestataire un effort de conversion d'une passion en profession (Dubois et Terral, 2011).

D'autres travaux ont montré que les pratiques professionnelles des prestataires sportifs n'étaient pas toutes semblables et étaient sous-tendues par différentes logiques d'action. C'est notamment le cas des travaux de Bouhaouala (2001, 2007, 2008) sur les dirigeants de petites et très petites entreprises du tourisme sportif, qui montrent que l'analyse de l'offre locale ne doit pas négliger les dimensions identitaires et la diversité des logiques d'action des professionnels. Ses travaux s'inscrivent dans la perspective wébérienne de l'action sociale qui renvoie au sens donné par les acteurs à leurs conduites. Il montre en effet que l'activité des dirigeants émane d'une diversité de logiques d'action pratiques inhérentes aux acteurs. Il s'agit alors de dépasser les approches de l'économie néoclassique, selon lesquelles la rationalité utilitaire constitue l'unique déterminant des comportements individuels, et les approches holistes de la sociologie, pour lesquelles ce sont les structures sociales qui sont déterminantes. Il est nécessaire de prendre en compte la diversité des motifs qui sont à l'origine des comportements sociaux et économiques des individus. Selon Weber (1995: 38 ), le motif est « un ensemble significatif qui semble constituer aux yeux de l'agent ou de l'observateur la raison significative d'un comportement». Les motifs peuvent s'exprimer sous forme de buts, d'objectifs, d'ambitions et sont directement liés à la vision du monde et à la conception que se font les acteurs de leurs activités. Cette vision du monde n'est pas entièrement héritée, mais elle est liée aux expériences sportives et professionnelles des acteurs. Bouahouala $(2001,2007,2008)$ montre également que les motifs et les visions du monde des prestataires sportifs produisent des logiques d'action spécifiques qu'il est possible de repérer à travers l'analyse des comportements et des choix économiques effectués par les dirigeants. Il a pu ainsi établir une typologie des entrepreneurs dirigeants d'entreprises de tourisme sportif : l'indépendant passionné (Bouhaouala et Chifflet, 2001), l'entrepreneur indépendant, le dirigeant gestionnaire et le conservateur patrimonial.

Dans la même perspective, Perrin-Malterre (2008) s'est intéressée aux logiques d'action des moniteurs de parapente sur le site du Puy-de-Dôme, en faisant référence aux travaux de Vachée et al. (2004) qui analysent les logiques d'action et les pratiques professionnelles des prestataires touristiques de sports de nature. L'auteure a ainsi mis en évidence trois logiques d'action professionnelles chez les moniteurs de parapente qui ont été qualifiés d'entrepreneurs, de passionnés et d'offreurs locaux.

En référence à des travaux menés en psychologie sociale, Vachée (2004) et Vachée et Raspaud (2003) analysent la manière dont les représentations de la nature des moniteurs d'escalade influencent leur logique d'offre sportive. La représentation est une forme de connaissance socialement élaborée et partagée qui permet aux individus de donner du sens au monde qui les entoure. Elle sert aussi à agir et influence les conduites sociales (Jodelet, 1984, citée par Vachée, 2001). Dans le cadre de cet article, l'offre sportive est saisie en fonction du discours produit à propos des comportements d'offre, et renseigne sur les caractéristiques et formes d'encadrement préconisées par les moniteurs, leurs orientations pédagogiques, ainsi que leurs façons de considérer les possibilités d'utilisation des espaces naturels avec les groupes qu'ils encadrent. Vachée (2001) met en évidence cinq modèles de représentations de la nature chez les moniteurs d'escalade qui ont une influence sur leur logique d'offre. Par exemple, les moniteurs «écologistes» sont centrés sur la recherche de sites naturels, sur la transmission du plaisir dans l'action de grimper et accordent, dans leurs discours éducatifs avec les personnes qu'ils encadrent, une large place à la sensibilisation au respect du milieu qui les accueille. À l'opposé, les moniteurs "progressistes» recherchent la rentabilité et la consommation immédiate dans le choix des sites fréquentés avec leurs groupes : les falaises doivent être accessibles, aménagées et permettre une mise en place rapide du matériel.

En référence à une approche structurelle des pratiques sociales, Corneloup et Bourdeau (2002) proposent un modèle d'analyse de la dynamique des cultures professionnelles dans les sports de nature. Ils prennent en compte les principes culturels des métiers sportifs de la montagne pour repérer les éléments structurant les logiques d'action professionnelles. La prise en compte de ces principes sert de référent pour aborder l'historique des métiers et en décoder les changements significatifs. Selon les auteurs, nous serions en présence de plusieurs générations de professionnels aux logiques d'action différentes qui se succèdent. La première génération de 
professionnels, celle des techniciens, s'ancre dans la culture moderne. Cette culture s'est affirmée au cours du XXe siècle (à partir des années 1930) avec la sportivisation de l’alpinisme et l'ouverture au métier de guide de haute montagne. La deuxième génération de professionnels, celle des offreurs de services sportifs, s'ancre dans la culture postmoderne. Cette culture a émergé au cours des années 1980 et va contribuer à transformer les pratiques sportives en activités commerciales. Selon Bourdeau et al. (2006) et Corneloup (2010), on assisterait à l'émergence d'une troisième génération de prestataires sportifs, celle des écodéveloppeurs récréatifs, qui s'inscrirait dans la culture transmoderne.

La transmodernité (Dussel, 2003; Grosfoguel, 2006; Luyckx-Ghisi, 2001; Rodriguez Magda, 2004) peut se saisir comme un mouvement qui souhaite dépasser les limites de la modernité en proposant un nouveau modèle de société que l'on pourrait qualifier de durable, mettant en exergue l'idée de finitude et de vulnérabilité du système moderne. L'émergence de la transmodernité est liée à des changements de fonctionnement de l'économie avec le développement d'une économie de la connaissance (Foray, 2000; Gorz, 2003, cités par Corneloup, 2011), et la progression du secteur de l'économie sociale et solidaire (Cohen, 2006, cité par Corneloup, 2011) et de l'économie verte. Selon les auteurs, on assisterait chez les acteurs à l'émergence de valeurs liées à la solidarité entre les hommes et les peuples, à la décroissance, à l'écologie et au respect. La déconstruction progressive du modèle hiérarchique pyramidal laisse place à un modèle d'organisation horizontal intégrateur. On assiste en effet à la montée en puissance de la démocratie participative (Gaudin, 2007, cité par Corneloup, 2011) et au passage de la politique publique à l'action publique (Lascoumes, 2007, cité par Corneloup, 2011).

Selon, Corneloup (2011), on assisterait, dans le domaine touristique et sportif, à l'émergence d'un style transmoderne, avec la montée progressive depuis les années 1990-2000 de pratiques qui s'inscrivent dans une démarche alternative. Ce style transmoderne se caractérise par une recherche d'immersion dans les profondeurs de la nature (Andrieu, 2011, cité par Corneloup, 2011), avec laquelle il s'agit de faire corps, par une approche métissée et un mélange des modalités de pratique, par la valorisation du voyage écologique associé à un réenchantement des lieux du quotidien. Il est aussi défini par la volonté de rompre avec la société de la vitesse et de l'instantanéité en privilégiant un déplacement lent et par une nouvelle culture du lien social, opérant un recentrage des pratiquants sur des valeurs essentielles, relevant plus de la sensibilité émotionnelle que du strict sensationnel.

Dans le cadre de ce travail, il s'agit de comprendre comment les changements issus de transformations sociétales, à savoir la montée de la transmodernité, affectent leurs pratiques professionnelles. Il s'agit notamment de mettre en évidence l'émergence d'une nouvelle catégorie de prestataires sportifs que l'on peut qualifier d'écodéveloppeurs récréatifs, pour reprendre la terminologie de Bourdeau et al. (2006), et surtout de caractériser leurs pratiques professionnelles.

Pour ce faire, nous choisissons d'écarter un cadre d'analyse qui s'appuierait sur une approche en termes de culture professionnelle, car cela renvoie à l'existence d'une profession établie
(Dubar et al., 2011). Par contre, dans le secteur du tourisme sportif, il s'agit davantage d'un "métier sans profession», comme c'est le cas pour les enseignants d'arts martiaux, «dans la mesure où aucun corps professionnel unifié ne vient réguler les conditions d'emploi et de travail» (Julhe et Haschar-Noé, 2010 : 26). Nous choisissons également d'écarter une approche en termes de représentation sociale, car elle nous paraît trop déterministe dans le sens où ce sont ces éléments qui vont influencer de manière univoque les conduites sociales des individus. Cependant, il ne s'agit pas non plus de privilégier un cadre d'analyse qui s'appuierait uniquement sur l'analyse stratégique de Crozier et Friedberg (1977), car elle considère que la conduite de l'individu s'inscrit dans une logique de pouvoir en étant principalement guidée par la poursuite d'intérêts propres, sans prendre en considération la construction historique et identitaire de l'acteur. Nous choisissons donc de privilégier un cadre d'analyse adapté de la sociologie des logiques d'action (Amblard et al., 1996 : 188), car cette approche permet de «lester l'acteur [...] de dimensions historiques, culturelles, qui sont trop rapidement exogénéisées par le raisonnement stratégique». Le concept de logique d'action permet également de dépasser les explications trop « déterministes» du comportement humain qui se limitent à associer l'action individuelle à telle ou telle caractéristique de l'acteur ou de son milieu.

Selon Amblard et al. (1996), une logique d'action est définie comme la combinaison du sens donné par l'individu à l'action entreprise et de la situation d'action dans laquelle il est placé. Dans le cadre de cette étude, nous retenons deux dimensions de la logique d'action professionnelle des prestataires sportifs: la dimension stratégique et la dimension sociohistorique, car la dimension stratégique de l'acteur doit être examinée non seulement à la lumière de l'interprétation qu'il fait de la situation, mais aussi en tenant compte de l'ensemble de ses expériences et de ses apprentissages antérieurs.

Dans la sphère du travail et de l'activité professionnelle, Amblard et al. (1996) soulignent que la dimension sociohistorique de l'acteur transparaît très concrètement dans le parcours professionnel individuel et dans l'expérience de travail. Ces éléments sont la résultante de l'activité antérieure en tant que «somme d'apprentissages qui sont autant d'éléments de structuration de manières d'être, de faire, d'analyser et d'investir les situations de travail» (Amblard et al., 1996 : 207). Par ailleurs, dans le domaine du tourisme sportif, l'encadrement contre rémunération d'un sport de nature demande des compétences techniques dans l'activité physique et sportive en question. Il s'agit donc de prendre en compte le passé sportif du prestataire. Ainsi les composantes de la dimension sociohistorique sont les suivantes :

- le parcours sportif du prestataire avec les activités pratiquées, l'ancienneté dans la pratique, le niveau et la fréquence de l'activité;

- son parcours de formation, que ce soit dans le domaine sportif et dans celui du tourisme;

- et son parcours professionnel et son expérience de travail.

En ce qui concerne la stratégie, celle-ci ne peut se résumer à un programme d'action prédéfini en fonction d'un objectif conscient poursuivi par l'acteur. Elle renvoie plutôt à une 
logique identifiable "après coup». Ainsi l'action dépend plus des ressources et des atouts dont l'acteur dispose et des relations dans lesquelles il est inséré que d'objectifs explicites et conscients qu'il se donne. En nous appuyant sur les travaux de Vachée et al. (2004) qui nous proposent une grille de lecture des stratégies professionnelles des prestataires sportifs de la nature et des acteurs du secours en montagne, nous retenons quatre composantes de la dimension stratégique en référence aux quatre pôles de l'action professionnelle telle qu'elle se construit dans le quotidien de la pratique :

- la logique pratique, qui s'intéresse à la manière dont le prestataire développe une relation d'échange avec la clientèle dans le cadre de la pratique;

- la logique sportive, qui étudie les modalités de pratique choisies, le rapport à la nature et les territoires professionnels utilisés par le prestataire;

- la logique organisationnelle, qui concerne les stratégies marketing choisies et les types de gestion développés par le professionnel;

- et la logique sociale, qui s'intéresse aux relations existantes entre le métier et la vie sociale et au rapport que le moniteur entretient avec l'espace local dans lequel se déroule l'activité professionnelle.

\section{Méthodologie}

Dans la perspective du courant de la sociologie des logiques d'action, nous avons employé une méthodologie compréhensive. "Comprendre, c'est entendre, admettre et apprendre de cette compréhension plutôt que d'expliquer à ces mêmes acteurs pourquoi ils ne savent pas ce qu'ils font» (Amblard et al., $1996: 169$ ). Nous avons accordé une grande importance à l'entretien avec les acteurs en considérant leur parole comme fondamentale. Nous sommes restés fidèles au contenu de leur parole sans chercher à discerner dans leurs propos une vérité cachée ni porter de jugement de valeur.

\section{Les acteurs interrogés}

Le choix des acteurs interrogés n'a pas été guidé par une recherche d'exhaustivité ou de représentativité de l'ensemble des activités sportives de nature proposées par les prestataires sur le territoire du Puy-de-Dôme. Ce choix a davantage été guidé par une volonté d'interroger des professionnels qui proposent des activités douces et de découverte du milieu, et qui s'inscrivent dans une démarche en lien avec la transmodernité. Pour cela, un contact a été pris avec la chargée de mission «sports de nature» du parc naturel régional des Volcans d'Auvergne pour être mis en contact avec des prestataires touristiques de sports de nature. Les premiers prestataires rencontrés nous ont ensuite mis en contact avec d'autres personnes ayant la même démarche que la leur. Ainsi, 10 prestataires sportifs âgés de 25 à 52 ans et implantés dans le massif du Sancy et dans la chaîne des Puys ont été interrogés : 4 accompagnateurs en moyenne montagne, 2 accompagnatrices de tourisme équestre, 3 moniteurs de parapente et 1 monitrice de ski.

\section{Le guide $d^{\prime}$ entretien}

Le guide d'entretien utilisé reprend les dimensions et les indicateurs des concepts définis dans le cadre d'analyse, à savoir la dimension sociohistorique de la logique d'action et la dimension stratégique. Il suit une logique qui part du parcours de formation et du parcours professionnel de l'acteur pour aboutir à une description de son activité professionnelle et de ses différentes composantes. Il s'agit enfin de replacer cette activité professionnelle dans une trajectoire de vie plus globale et de voir les modalités de son articulation avec une vision du monde et de la société.

\section{Le déroulement des entretiens}

Un entretien semi-directif (Blanchet, 1985) a été réalisé avec chaque prestataire sportif. Ces entretiens, réalisés sur une période allant de fin mars à début septembre 2011, ont une durée moyenne de 80 minutes. Ils se sont déroulés en personne, soit sur le lieu de travail des professionnels (cinq entretiens), soit à leur domicile (trois entretiens), soit dans un café (deux entretiens). Avant de débuter l'entretien, l'autorisation d'enregistrer les propos a été demandée aux prestataires. L'ensemble d'entre eux a accepté cette condition, ce qui fait que tous les entretiens ont pu être enregistrés à l'aide d'un dictaphone.

\section{L'analyse des données}

À partir des entretiens semi-directifs intégralement retranscrits, une analyse qualitative a été réalisée. Plus précisément, l'analyse thématique a été privilégiée. Elle consiste à "procéder systématiquement au repérage, au regroupement et, subsidiairement, à l'examen discursif des thèmes abordés dans un corpus» (Paillé et Mucchielli, 2003 : 162). L'unité thématique est « un noyau de sens repérable en fonction de la problématique et des hypothèses de la recherche» (Blanchet et Gotman, 2005 : 98). Les thèmes ont été sélectionnés en fonction du cadre théorique et ont constitué un cadre stable d'analyse de tous les entretiens. Il s'agit donc d'une procédure close de catégorisation (Ghiglione et Matalon, 1991), dans le sens où les thèmes ont été définis préalablement à l'analyse. Dans un premier temps, l'analyse a porté sur chaque entretien afin de déterminer le processus de construction de chaque logique d'action professionnelle chez les prestataires. Dans un deuxième temps, une analyse comparative des données a permis de repérer les points communs entre les acteurs et de dégager les caractéristiques de la logique d'action professionnelle de l'écodéveloppeur récréatif.

\section{Résultats \\ Des parcours professionnels liés à une passion pour les sports de nature}

Tous les acteurs interrogés ont une longue pratique d'activités sportives de nature, parfois depuis leur plus jeune âge. Pour la monitrice, «le ski, [elle] en fai[t] depuis toute petite». Un accompagnateur en moyenne montagne explique que «depuis l'âge de quatre ans, [il] faisai[t] des randonnées d'une semaine tous les étés avec [s]es parents». Pour sa part, une accompagnatrice de tourisme équestre a «toujours aimé les chevaux et [a] eu la chance d'habiter près d'un centre équestre et de pouvoir commencer très jeune». Un moniteur de parapente explique qu'il était «un petit peu turbulent. Un jour, un copain de $[s]$ on père lui a dit : "Donne-nous ton fils, on va 
le calmer". [Il s'est] accroché sous un delta, ça [1]'a calmé, et depuis, [il n'a] pas arrêté l'activité. Au contraire, [il] en [a] fait [s]on métier.» Très vite, pour ces acteurs s'est posée la question de faire de leur passion une profession et d'encadrer des personnes dans l'apprentissage moteur. Or, en France, selon la Loi sur le sport de 1984 modifiée par celle de 2000, les acteurs de l'offre ont l'obligation d'être titulaire d'un Brevet d'État d'éducateur sportif dans la discipline qu'ils encadrent. C'est ainsi que les trois accompagnateurs en moyenne montagne, la monitrice de ski, une accompagnatrice de tourisme équestre et un moniteur de parapente ont rapidement passé le Brevet d'État d'éducateur sportif pour pouvoir encadrer l'activité. "Je pratique l'activité depuis 1982, et j'enseigne delta et parapente depuis 1986.» Ce glissement rapide vers la professionnalisation peut être interprété comme «l'expression d'une passion pour la pratique [mais aussi] comme le signe d'une vocation pour l'enseignement ", expliquent Julhe et HascharNoé (2010: 12).

Un moniteur de parapente et une accompagnatrice de tourisme équestre sont venus à l'activité qu'ils encadrent plus tardivement. Cette dernière, dans le cadre de ses études de BTS tourisme, a fait «un stage en centre équestre alors qu['elle] ne montai[t] pas du tout à cheval. Le moniteur [1]'a fait monter un peu, mais [elle] étai[t] nullissime. Mais pendant le stage, il a vu qu['elle] progressai[t] énormément. Donc [elle est] repartie en stage chez lui, [elle a] passé les galops. Et après, [elle est] partie dans le Cantal faire un autre stage à cheval.» Le moniteur de parapente, pour sa part, est «venu au parapente parce qu['il] avai[t] des copains d'escalade qui en faisaient [...] Dès qu['il a] eu les sous, [il s'est dit] : "Tiens, il faudrait que j'essaye ça". [Il a] essayé et ça l'a emporté sur le reste.» Ce moniteur a donc décidé de quitter son emploi dans l'éducation nationale pour passer le Brevet d'État d'éducateur sportif et devenir prestataire d'activités sportives : «Au départ j'étais enseignant [...] Mais j'étais en contradiction, en porte-à-faux entre ce que je sentais, ce que je voyais, mes idées et puis mon enseignement; un écart de plus en plus grand. J'ai donc décidé de me reconvertir, de devenir professionnel du sport en passant mon Brevet d'État.»

Ainsi, tous les prestataires sportifs interrogés sont passés d'un statut de pratiquants consommateurs à celui d'offreurs de services sportifs. Ils ont donc été amenés à transformer une passion pour le sport en profession durable (Lima et Mossé, 2010) afin de pouvoir allier plaisir et travail, comme c'est le cas pour les enseignants d'art martiaux (Juhle et HascharNoé, 2010) et pour les pratiquants de l'équitation (Chevalier et Dussart, 2002). Leur activité professionnelle est ainsi une opportunité pour eux d'assouvir leur passion pour le sport. Comme l'expliquent Bouhaouala et Chifflet (2001 : 65), la passion doit s'envisager comme "l'intérêt que porte un ensemble d'acteurs au sport, à la nature et au plaisir qu'ils en tirent. En ce sens, c'est une motivation sociale (partagée) pouvant déterminer certains comportements sociaux et économiques». On peut également constater qu'hormis une des accompagnatrices de tourisme équestre, aucun autre prestataire n'a de formation dans le domaine du tourisme ou de la gestion d'entreprise. Cependant, grâce à leur ancienneté dans la pratique de sports de nature, ils font preuve de très bonnes compétences sportives, et ce sont sur ces compétences que s'appuie leur parcours professionnel. À l'instar des enseignants d'art martiaux, ils se placent «à l'interface entre compétences techniques, acquises au cours de leur socialisation sportive, et compétences gestionnaires, développées de manière beaucoup plus autodidactes» (Julhe et Haschar-Noé, $2010: 25$ ).

Après avoir étudié le parcours sportif et professionnel des prestataires interrogés, il s'agit maintenant de reconstruire leurs stratégies professionnelles afin d'analyser la manière dont les changements issus des transformations sociétales affectent leur pratique et participent à l'émergence d'une nouvelle logique d'action professionnelle.

\section{L'émergence d'une nouvelle logique d'action professionnelle}

Afin d'analyser les stratégies professionnelles des acteurs interrogés, nous allons étudier les quatre pôles de l'action professionnelle telle qu'elle se construit dans le quotidien de la pratique.

\section{La logique pratique}

Pour l'ensemble des prestataires interrogés, l'accueil et l'échange avec les clients est un aspect primordial de la prestation. "Pour moi, l'accueil, c'est primordial», commente un accompagnateur en moyenne montagne. "Après, c'est la disponibilité. Moi, je crois que la disponibilité, les clients adorent.» Selon un autre accompagnateur, «prendre le temps d'être à l'écoute des gens, c'est une qualité essentielle dans le tourisme aujourd'hui et qui se démarque complètement des grosses structures». Dans la même perspective, un des moniteurs de parapente souhaite développer des stages sur plusieurs jours car, selon lui, «l'enseignement en stage, c'est des rapports qui s'installent dans la durée. Humainement, c'est plus riche». Il s'agit d'une volonté de réhumanisation de la relation prestataire/client qui se traduit par de l'écoute, de l'échange et de l'attention portée à l'autre. Cela se manifeste aussi par l'adaptation au rythme du client : "On prend son temps, il n'y a pas d'échéance dans la journée, on évolue selon les envies des gens. Ce qui est important, c'est plutôt le bienêtre, la découverte du milieu.» La monitrice de ski explique : «Ici, ce n'est pas l'usine comme dans les grandes stations. Si le cours dure un quart de plus, ce n'est pas grave. On n'est pas là pour enchaîner.» Ainsi, la coproduction et le copilotage des pratiques déjà présents dans la culture postmoderne se renforcent. On observe une volonté d'impliquer et de faire participer le client à être pleinement acteur de ce qu'il vient vivre.

Le moment d'échange avec le client lors de la prestation est également un moment de transmission qui sert à faire passer des messages autour de la notion de développement durable. Comme le montre en effet Bessy (2008), les sports de nature sont en mesure de s'inscrire dans les trois dimensions du développement durable et de répondre à des enjeux de développement économique, de cohésion sociale et de préservation de l'environnement. Ces enjeux sociaux et environnementaux sont mis en avant par les acteurs dans leurs échanges avec la clientèle. Ainsi, ils n'hésitent pas à sensibiliser, voire à éduquer leur client à la dimension écologique et à la solidarité. Selon une accompagnatrice de tourisme équestre : «Moi, j’aime 
transmettre le côté amoureux de cette nature qui est préservée; le respect de la nature dans laquelle j'évolue au quotidien. Le respect des gens aussi.» Un des accompagnateurs en moyenne montagne nous explique que «le passage ici doit permettre de faire en sorte que les gens fassent attention après. Il faut réussir à faire évoluer le comportement des uns et des autres, puis le changement se fera au fur et à mesure». Ces prestataires se situent dans la perspective des mouvements d'éducation à l'environnement (Pineau et al., 2005) qui proposent de renouveler notre relation à la nature, celle-ci ayant «une valeur écologique et autonome [en acquérant] un droit ontologique à l'existence» (Kalaora, 2001 : 592). «On quitte dans cette perspective le projet cartésien et humaniste de la modernité lorsqu'il s'agit de faire corps avec la nature en limitant au maximum son empreinte sur elle et en modifiant les catégories de lecture du prédateur prométhéen et contemplatif et celles du free-rider à la recherche d'une nature ludique » (Corneloup, 2011:6).

\section{La logique sportive}

Les valeurs de l'effort et de la rigueur de la culture moderne, ainsi que le traitement entrepreneurial des pratiques qui valorisent l'émotion et les sensations dans la culture postmoderne laissent la place à des pratiques plus douces, mais qui demandent un investissement de la part du client. Un accompagnateur en moyenne montagne l'explique : "Je cherche à faire quelque chose qui soit ludique, mais qui demande un peu d'investissement personnel; aller chercher au fond de soi qui l'on est...» Les pratiques proposées sont aussi axées sur la découverte de l'environnement proche. Une accompagnatrice de tourisme équestre nous explique que «la balade de deux heures, même si on n'est pas trop gaillard à cheval, on se met la tête à l'envers, car on a l'impression de partir super loin et, en fait, on est juste à côté. Donc, tout de suite, on est en contact de la nature. Et il y a une variété de paysages : on est en montagne et après on bascule dans une forêt de hêtres. » Ainsi, les prestataires privilégient les activités de proximité. Ils répondent ainsi à une certaine demande de touristes qui s'interrogent sur le bien-fondé de s'envoler au bout du monde pour retrouver le même univers qu'au coin de la rue qui et sont à la recherche d'un tourisme alternatif de proximité (Michel, 2010). Pour eux, il s'agit avant tout de découvrir l'environnement local et le patrimoine. C'est ainsi que les accompagnateurs en moyenne montagne répondent à cette demande, en axant leurs séjours sur la découverte du patrimoine naturel et culturel des monts d'Auvergne, de lacs en cascade et des montagnes aux volcans. «Je vais expliquer les fleurs, le paysage. S'il y a un paysan à côté, on s'arrête, on va blablater avec lui.» L'un d'entre eux, passionné de volcanologie, a développé un séjour sur la découverte des phénomènes volcaniques du passé pour mettre les touristes en relation avec les volcans actifs de la planète. Un des moniteurs de parapente «se sent comme une sorte d'agent touristique». Il explique ainsi à ses clients ce qu'ils peuvent visiter dans la région. C'est ainsi que se développe un tourisme local (Amirou et Bachimon, 2000) dans lequel le client s'affirme comme un «consom'acteur du voyage », dans le respect du lieu et de ses habitants, et non plus comme un consommateur passif de circuits touristiques.

\section{La logique organisationnelle}

La logique organisationnelle des prestataires est guidée par leur conception de l'activité et orientée vers un désir d'indépendance. Un moniteur de parapente explique qu'après avoir travaillé dans une structure où ils étaient une dizaine de salariés, il a décidé de créer sa propre structure : «Une structure beaucoup moins lourde, avec beaucoup moins de personnel, avec pour motivation l'envie de travailler d'une manière indépendante, d'être son propre patron.» Tous les acteurs interrogés ont le statut de travailleur indépendant et conservent ce statut, même s'ils sont regroupés avec d'autres, comme c'est le cas pour la monitrice de ski qui enseigne dans une école de ski. Cependant, ce désir d'indépendance ne se traduit pas non plus par un isolement, car ils font preuve d'une capacité à savoir s'entourer et à s'insérer dans des réseaux locaux.

On observe également un rejet de la culture libérale et marketing des prestataires postmodernes pour lesquels la nature est perçue comme une ressource externe permettant de réaliser du profit. Les prestataires interrogés font le choix d'un développement modeste et limité par peur de perdre leurs valeurs et leur personnalité du fait de l'augmentation du nombre de clients. Ainsi, l'ensemble des prestataires interrogés ne souhaite pas développer leur activité davantage : «Pour ma part, c'est bien comme ça. Je ne suis pas sûre que, si je développais à fond, ce serait aussi bien.» On retrouve ici une des caractéristiques des moniteurs indépendants passionnés décrits par Bouhaouala et Chifflet (2001) et dont les comportements économiques restent guidés par leur passion pour l'activité sportive. Ainsi, «ils maintiennent leur entreprise et leur volume d'activités au niveau qui leur permet l'indépendance et évite[nt] l'assouvissement de leur passion face aux pressions économiques, velléités de rachat et tendances de croissance» (Bouhaouala, $2007: 8$ ). On constate chez ces prestataires une réelle volonté de ne pas rentrer dans un système mercantile où l'argent serait une fin en soi. C'est le cas d'un des moniteurs de parapente qui a quitté son emploi de fonctionnaire : «Ma carrière professionnelle de parapente, moi, c'était pour mieux vivre, pour me sentir mieux dans ma tête; même avoir moins de fric, puisque, quand j'ai débuté, j'avais quatre fois moins de fric que quand $j$ 'avais ma paye de fonctionnaire.» Ainsi, l'argent n'est pas la finalité, mais c'est la qualité de vie qui prime.

\section{La logique sociale}

On constate chez les acteurs un ancrage dans le territoire où se déroule leur activité professionnelle. En effet, tous les prestataires interrogés habitent dans le massif du Sancy ou dans la chaîne des Puys et manifestent un attachement pour leur massif. Un moniteur de parapente en atteste : «Je suis fier de ma région. J'aime bien travailler ici : le Puy-de-Dôme avec son antenne, quand on arrive de Thiers et qu'on le voit, on se sent chez soi.» Pour la monitrice de ski, «c'est une imprégnation du paysage, du village, du clocher. L'attachement, il est viscéral.» Ces prestataires ne sont pas pour autant retirés du monde. Ils sont inscrits dans des réseaux d'acteurs touristiques et participent parfois à la vie locale. Ainsi la majorité d'entre eux sont en contact et ont créé des liens avec d'autres acteurs touristiques qui ont des activités complémentaires 
aux leurs (comme des hébergeurs, par exemple). Par ailleurs, trois prestataires sont élus dans leur commune, dont la monitrice de ski qui est également élue à la communauté de communes du Sancy : «La communauté de communes du Sancy, c'est une grosse communauté qui va de l'avant. Les autres communes, elles te tirent vers le haut; tu es obligé de suivre. De bosser sur des projets avec eux, je trouve ça génial». Un des accompagnateurs en moyenne montagne fait partie du réseau départemental École et Nature, réseau qui fédère les acteurs impliqués dans l'éducation à l'environnement : «C'est un réseau qui permet de rencontrer d'autres personnes, de discuter et d'échanger sur ce qu'on fait comme action d'éducation à l'environnement.» Il est donc en lien avec d'autres acteurs du territoire issus d'horizons divers et pas seulement du monde sportif. Ainsi, ces prestataires veulent être plus actifs dans la gestion de leur quotidien et souhaitent participer aux activités associatives et politiques de leur lieu de vie. On retrouve ici les écrits de Ray et Anderson (2001) sur les créatifs culturels qui envisagent des implications associatives et politiques dans leur vie quotidienne.

Ainsi, ces prestataires souhaitent redonner du sens et de la valeur à l'accueil et à l'échange avec le client, tout en prenant leur temps. Ils favorisent les pratiques de proximité à la découverte d'un environnement riche et varié. Leur objectif est de faire prendre conscience au client qu'ils accompagnent la beauté et la fragilité d'une nature qui nécessite d'être respectée. Ils ont fait le choix d'un développement limité pour rester en accord avec leurs valeurs et conserver la richesse de l'échange. Enfin, ils font preuve d'un fort attachement au lieu qui se traduit parfois par une implication dans la vie locale.

\section{Un renouvellement des pratiques professionnelles}

Il apparaît que nous sommes en présence d'un renouvellement des pratiques professionnelles dans les métiers touristiques liés à l'encadrement des sports de nature avec l'émergence de prestataires qui souhaitent s'engager dans l'écodéveloppement récréatif. Les formes pédagogiques et relationnelles, les pratiques proposées, les formes organisationnelles choisies et les stratégies marketing employées, ainsi que la relation avec le local, le lieu et la nature s'inscrivent dans un autre projet professionnel que les techniciens du sport et les offreurs de services sportifs. Toutefois, on peut se demander si le positionnement des acteurs interrogés n'est pas uniquement un argument marketing pour surfer sur la vague de la prise de conscience écologique et ainsi se distinguer de la concurrence. Il nous semble que l'offre nouvelle qu'ils proposent s'inscrit dans la culture transmoderne et s'appuie sur des valeurs personnelles qu'ils transposent dans leur activité professionnelle, même si cela mériterait d'être vérifié par des observations participantes. Cette offre nouvelle qu'ils proposent se caractérise par une approche non prioritairement sportive de la prestation en mettant davantage l'accent sur la préservation de l'environnement et la découverte du patrimoine local. Précisons que cette notion de découverte se détache de la simple action de visiter au profit d'une démarche compréhensive et d'apprentissage par la sensibilisation, voire l'éducation. Dans cette perspective, les prestataires privilégient les activités douces et de proximité dans une logique d'adaptation : il s'agit en effet pour eux de construire une offre à partir des ressources locales (paysage, patrimoine, gastronomie...). Par ailleurs, la priorité est donnée à l'aspect personnel et relationnel de la prestation dans une logique de partage. Il ne s'agit plus d'une simple transmission de connaissances ou de compétences, mais davantage d'un échange réciproque puisque, s'ils sensibilisent leur clientèles, ils sont aussi prêts à recevoir des conseils en retour. Cela demande au prestataire d'être disponible et à l'écoute et de s'adapter dans une certaine mesure à la personnalité de son client, ce qui participe à une réhumanisation de la relation marchande. Dans ce cadre, les prestataires se situent davantage dans une logique de fidélisation de la clientèle plutôt que dans une stratégie marketing offensive de conquête de nouveaux marchés et de croissance économique. Enfin, l'offre proposée est liée à une inscription des acteurs dans le développement local avec la construction de réseaux d'acteurs touristiques ayant des activités complémentaires et un ancrage territorial qui se traduit par une implication dans des activités associatives ou politiques du territoire.

Pour terminer, on peut se demander si les écodéveloppeurs récréatifs ne sont pas en tous points identiques aux «indépendants passionnés» étudiés par Bouahouala et Chifflet (2001). Il apparaît dans nos résultats que, si ces deux catégories d'acteurs ont des attributs similaires et proches, ils s'en distinguent, car ils ne suivent pas une logique d'action tout à fait identique. En effet, les indépendants passionnés sont passionnés par leur métier, et sont eux aussi à la recherche de compromis et d'harmonisation entre leur action professionnelle et leur vision personnelle. Cependant, il semble que leur comportement soit essentiellement guidé par une logique d'action hédoniste avec une démarche productive centrée sur soi, dans le souci d'un épanouissement personnel. Autrement dit, ils semblent témoigner d'une logique plutôt individualiste et sont moins sensibles aux questions de société plus globales telles que les enjeux du développement durable, ou du moins ils ne cherchent pas à les inclure d'une manière ou d'une autre dans leur profession. Au contraire, pour les écodéveloppeurs récréatifs, le plaisir ne vient pas seulement du fait de pouvoir pratiquer un métier passionnant et de transmettre des compétences techniques liées à une activité sportive. C'est aussi sensibiliser les visiteurs à d'autres thématiques beaucoup plus larges et variées comme l'écologie, mais aussi la solidarité et le respect, avec une volonté de faire évoluer les comportements vers un meilleur respect du milieu naturel et dans l'optique d'un mieux «vivre-ensemble» et en société. De plus, les écodéveloppeurs récréatifs, à la faveur d'une implication locale marquée par leur participation à des activités sociales, politiques ou associatives, contribuent à s'ancrer territorialement, sans pour autant observer un repli sur le local comme Bouhaouala et Chifflet (2001) ont pu le constater chez les indépendants passionnés du Vercors.

Il s'agit tout de même de nuancer nos propos car, si la majorité des prestataires interrogés se retrouve dans la logique d'action de l'écodéveloppeur récréatif, deux acteurs s'en éloignent. Il s'agit d'un accompagnateur en moyenne montagne et d'un moniteur de parapente. En effet, même si ces derniers partagent avec les autres acteurs des valeurs de respect de l'environnement et de la nature, ou encore de la convivialité et 
de l'échange humain, ils ne semblent pas concernés par des problèmes de société plus globaux comme les questions d'écologie, de solidarité sociale ou de vivre-ensemble. Leur implication se limite au niveau local ou territorial : la chaîne des Puys pour le moniteur de parapente et le massif du Sancy pour l'accompagnateur en moyenne montagne. Ils sont plus préoccupés par l'avenir du tourisme dans leur territoire et la perduration de leur activité professionnelle que de faire des liens entre des problématiques globales qui traversent la société et leur intervention au niveau local. On observe également que s'ils sont attachés au lieu dans lequel ils exercent leur activité professionnelle, ils ne sont pas actifs dans des réseaux associatifs du territoire et n'exercent pas de responsabilité politique. Ils ont tout de même tissés des liens informels avec d'autres prestataires, mais uniquement des prestataires sportifs. Ainsi, ces acteurs ont davantage une démarche productive centrée sur eux et font preuve d'un certain repli sur le local, comme c'est le cas pour les indépendants passionnés.

\section{Conclusion}

En conclusion, nous pouvons affirmer que les changements culturels qui traversent la société et qui sont marqués par la montée de la transmodernité se traduisent dans le domaine du tourisme sportif et des sports de nature par des pratiques professionnelles renouvelées et renvoient à l'émergence d'une nouvelle logique d'action professionnelle. Les écodéveloppeurs récréatifs s'inscrivent en effet dans un autre projet professionnel que les techniciens du sport et les offreurs de services sportifs. Ils proposent des pratiques sportives douces axées sur la découverte de l'environnement proche, dans lequel la nature n'est pas un simple cadre de pratique. Dans le cadre de leur prestation, ils souhaitent redonner du sens et de la valeur à l'accueil et à l'échange avec le client. Ils ont fait le choix d'un développement limité et ils font preuve d'un fort attachement au lieu. Il semble alors intéressant de se pencher sur les compétences nécessaires pour ces prestataires, la compétence pouvant «être entendue non seulement comme un ensemble de connaissances professionnelles et de savoir-faire en situation de travail mais aussi comme un ensemble de propriété sociales des individus et un "savoir-être" au sens d'un ensemble de dispositions acquises socialement» (Gasparini et Pichot, 2011 : 18). Pour les prestataires sportifs, même si la compétence technique et sécuritaire reste au cœur de leur pratique professionnelle, il s'agit pour eux de développer de nouvelles compétences, et plus précisément (Corneloup, 2011) :

- une compétence écologique, et notamment la connaissance des milieux et des écosystèmes permettant de sensibiliser la clientèle;

- des compétences humaines et relationnelles en favorisant l'échange, la communication, et l'empathie avec le client;

- des compétences pour monter des projets collectifs et participer au développement du territoire;

- et enfin une compétence créative pour produire des innovations récréatives.

La question qui se pose alors est de savoir comment peuvent se construire ces compétences. En effet, la première relève davantage d'un ensemble de connaissances pouvant être acquises par le biais de formations initiales ou continues.
Cependant, les trois autres relèvent davantage du savoir-faire et du savoir-être et demandent à être acquises de manière beaucoup plus autodidacte au cours de la socialisation de l'individu et tout au long de son parcours sportif, de formation et professionnel.

\section{Références}

AMBLARD, Henri; Philippe BERNOUX; Gilles HERREROS et YvesFrédéric LIVIAN (1996) Les nouvelles approches en sociologie des organisations, Paris : Seuil. 291 pages.

AMIROU, Rachid et Philippe BACHIMON (2000) Le tourisme local, une culture de l'exotisme, Paris : L'Harmattan. 238 pages.

ANDRIEU, Bernard (2011) Un goût de terre. Vers la cosmosensation, Atlantica: Paris, cité dans CORNELOUP, Jean (2011) «La forme transmoderne des pratiques récréatives de nature», Développement durable et territoires, vol. 2, nº 3, <http://developpementdurable. revues.org/9107>, consulté le 15 janvier 2012.

BESSY, Olivier (2008) Sport, loisirs, tourisme et développement durable des territoires, Voiron : Presses universitaires du sport. 178 pages.

BLANCHET, Alain (1985) L'entretien dans les sciences sociales : l'écoute, la parole et le sens, Paris : Dunod. 289 pages.

BLANCHET, Alain et Anne GOTMAN (2005) L'enquête et ses méthodes : l'entretien, Paris : Armand Colin. 128 pages.

BOUHAOUALA, Malek (2001) «Relations inter-entreprises dans un marché local : le cas des PE-TPE du tourisme sportif en Vercors", Espaces et Sociétés, $\mathrm{n}^{\circ}$ 105-106, p. 229-251.

BOUHAOUALA, Malek (2007) «Micro-mentalités et logiques d'action des entrepreneurs dirigeants de petites entreprises», Revue Internationale PME, vol. 20, n 2, p. 123-149.

BOUHAOUALA, Malek (2008) Management de la petite entreprise des loisirs sportifs. Une approche socioéconomique, Bruxelles : De Boeck. 158 pages.

BOUHAOUALA, Malek et Pierre CHIFFLET (2001) «Logiques d'action des moniteurs des sports de pleine nature : entre passion et profession ", STAPS, nº 56, p. 61-74.

BOURDEAU, Philippe (2007) Les sports d'hiver en mutation : crise ou révolution géoculturelle?, Hermes Science publications, Paris : Lavoisier. 250 pages.

BOURDEAU, Philippe; Jean CORNELOUP et Pascal MAO (2006) «Cultures, espaces et métiers sportifs de la montagne : changements et enjeux d'avenir ", DANS BOURDEAU, Philippe (sous la direction de), La montagne terrain de jeux et d'enjeux, p. 11-28. Fournel : L’Argentière la Bessé.

CHARLES, Lionel et Bernard KALAORA (2007). «De la protection de la nature au développement durable : vers un nouveau cadre de savoir et d'action?», Espaces et Sociétés, no 130, p. 121-133.

CHEVALIER, Vérène et Brigitte DUSSART (2002) «De l'amateur au professionnel : le cas des pratiquants de l'équitation ", L'année sociologique, vol. $52, \mathrm{n}^{\circ} 2$, p. 459-476.

COHEN, Daniel (2006) Trois leçons sur la société post-industielle, Paris : Seuil, cité dans CORNELOUP, Jean (2011) «La forme transmoderne des pratiques récréatives de nature», Développement durable et territoires, vol. 2, $\mathrm{n}^{\circ} 3,<\mathrm{http}: / /$ developpementdurable. revues.org/9107>, consulté le 15 janvier 2012.

CORNELOUP, Jean (2009) «Dynamique culturelle et loisirs sportifs de nature», DANS FERREOL, Gilles (sous la direction de), Représentations corporelles et loisirs sportifs, Bruxelles : Intercommunications.

CORNELOUP, Jean (2010) «Innover par la forme transmoderne», DANS CORNELOUP, Jean (sous la direction de), Créativité et innovation dans les loisirs sportifs de nature, p. 72-98. Fournel : L’Argentière la Bessé. 
CORNELOUP, Jean (2011) «La forme transmoderne des pratiques récréatives de nature», Développement durable et territoires, vol. $2, \mathrm{n}^{\circ} 3,<\mathrm{http}: / /$ developpementdurable. revues.org/9107>, consulté le 15 janvier 2012.

CORNELOUP, Jean et Philippe BOURDEAU (2002) «Culture professionnelle et métiers du tourisme sportif de la montagne», Téoros, vol. 20, no 3, p. 32-44.

CROZIER Michel et Erard FRIEDBERG (1977) L'acteur et le système. Les contraintes de l'action collective, Paris : Seuil. 500 pages.

DUBAR, Claude, Pierre TRIPIER et Valérie BOUSSARD (2011) Sociologie des professions, Paris : Armand Colin. 376 pages.

DUBOIS, Fanny et Philippe TERRAL (2011) «De l'amateur sportif au dirigeant d'une petite entreprise. Le tourisme sportif de pleine nature», Travail et Emploi, $\mathrm{n}^{\circ}$ 126, <http://travailemploi.revues.org/5229>, consulté le 15 janvier 2012.

DUSSEL Enrique (2003) L'éthique de la libération. À l'ère de la mondialisation et de l'exclusion, Paris : l'Harmattan. 265 pages.

FORAY, Dominique (2000) L'économie de la connaissance, Paris : La Découverte. 125 pages.

GASPARINI, William et Lilian PICHOT (2011). «Le travail dans le sport : genèse d'un objet et perspectives interdisciplinaires», DANS GASPARINI, William et Lilian PICHOT (sous la direction de), Les compétences au travail : sport et corps à l'épreuve des organisations, p. 7-35. Paris : l'Harmattan.

GAUDIN, Jean-Pierre (2007) La démocratie participative, Paris : Armand Colin, cité dans CORNELOUP, Jean (2011) «La forme transmoderne des pratiques récréatives de nature», Développement durable et territoires, vol. 2, n $3,<$ http://developpementdurable. revues.org/9107>, consulté le 15 janvier 2012.

GHIGLIONE, Rodolphe et Benjamin MATALON (1991) Les enquêtes sociologiques : théorie et pratique, Paris : Armand Colin. 301 pages.

GIRARD, Alain (2009) «Faut-il raccorder une théorie à moyenne portée de la post-touristicité à une théorie générale de la post-modernité?», Communication présentée au colloque Fins et confins du tourisme, Grenoble, 26-27 mai.

GORZ, André (2003) L'immatériel, Galilée : Paris, cité dans CORNELOUP, Jean $(2011)$ «La forme transmoderne des pratiques récréatives de nature», Développement durable et territoires, vol. $2, \mathrm{n}^{\circ} 3,<\mathrm{http}: / /$ developpementdurable. revues.org/9107>, consulté le 15 janvier 2012.

GROSFOGUEL, Ramon (2006) «Les implications des altérités épistémiques dans la redéfinition du capitalisme global», Multitudes, vol. 3, $\mathrm{n}^{\circ}$ 26, p. 51-74.

JODELET, Denise (sous la direction de), (1984) Les représentations sociales, Paris : PUF. 447 pages.

JULHE, Samuel et Nadine HASCHAR-NOE (2010) «Trajectoires et stratégies professionnelles dans le secteur de l'animation sportive : le cas des enseignants d'arts martiaux", Sociétés contemporaines, $\mathrm{n}^{\circ}$ 77, p. 7-29.
KALAORA, Bernard (2001) «À la conquête de la pleine nature», Ethnologie française, vol. XXXI, nº 4, p. 591-597.

LASCOUMES, Pierre (2007) Sociologie de l'action publique, Paris : Armand Colin, cité dans CORNELOUP, Jean (2011) «La forme transmoderne des pratiques récréatives de nature», Développement durable et territoires, vol. $2, \mathrm{n}^{\circ} 3,<\mathrm{http}: / /$ developpementdurable. revues.org/9107>, consulté le 15 janvier 2012.

LIMA, Léa et Philippe MOSSE (2010) «Conclusion générale. Concilier passion et profession : une approche conventionnaliste», DANS LIMA, Léa et Philippe MOSSE (sous la direction de), Le sport comme métier? Les STAPS des études à l'emploi, p. 135-148. Toulouse : Octarès.

LUYCXK-GHISI, Marc (2001) Au-delà de la modernité, du patriarcat et du capitalisme, Paris : L'Harmattan. 216 pages.

MICHEL, Franck (2010) «À la recherche d'un tourisme alternatif de proximité», Espaces, n 285, p. 14-28.

PAGET, Elodie; Jean-Pierre MOUNET et Alice GHUILLON (2007) «Les petites entreprises prestataires de sport et de tourisme et leurs dirigeants : spécificités et stratégies", REMS, n ${ }^{\circ}$ 17, p. 2-13.

PAGET Élodie et Jean-Pierre MOUNET (2010) «Flexibilité de l'entreprise et implication des employés», Travail et Emploi, no 122, <http://travailemploi.revues.org/1539>, consulté le 15 janvier 2012.

PAILLE, Pierre et Alex MUCCHIELLI (2003) L'analyse qualitative en sciences humaines et sociales, Paris : Armand Colin. 424 pages.

PERRIN-MALTERRE, Clémence (2008) «Le parapente au sommet du Puy de Dôme. Analyse d'un territoire touristique et sportif », Loisir et Société, vol. 30, n 2, p. 443-464.

PINEAU Gaston; Dominique BACHELART et Dominique COTTEREAU (2005) Habiter la Terre : écoformation terrestre pour une conscience planétaire, Paris : L'Harmattan. 292 pages.

RAY, Paul et Sherry Ruth ANDERSON (2001) L'émergence des créatifs culturels, Gap : Yves Michel. 512 pages.

RECH, Yohann et PAGET, Elodie (2012) «Les temporalités du travail touristique», Socio-logos. Revue de l'association française de sociologie, $\mathrm{n}^{\circ} 7$, $<$ http://socio-logos.revues.org/2674>, consulté le 15 janvier 2012.

RODRIGUEZ MAGDA, Rosa-Maria (2004) Transmodernidad, Barcelone : Anthropos. 22 pages.

VACHÉE, Cécile (2004) «Représentations et imaginaires de la nature en escalade», Cahiers de l'imaginaire, n ${ }^{\circ}$ 20. P. 20-30.

VACHÉE, Cécile; Jean CORNELOUP et Bastien SOULÉ (2004) «Logiques professionnelles et référents culturels dans les métiers sportifs de montagne», DANS SOBRY, Claude (sous la direction de), Le tourisme sportif, p. 161-189. Villeneuve d'Ascq : Presses universitaires du Septentrion.

VACHÉE, Cécile et Michel RASPAUD (2003) «Représentation de la nature et logiques d'offres chez les moniteurs d'escalade», Sciences et Motricité, $\mathrm{n}^{\circ} 48$, p. 29-47.

WEBER, Max (1995) Économie et société, Paris : Plon. 411 pages. 